\title{
Novel Clue to Locate the Conduction Gap on the Pulmonary Vein Isolation Ablation Line
}

\author{
Hai-yang Xie ${ }^{1}$, Xiao-gang Guo ${ }^{2}$, Jian-du Yang ${ }^{2}$, Hui-qiang $\mathrm{Wei}^{3}$, Bin Luo ${ }^{3}$, Yan-qiao \\ $\mathrm{Chen}^{2}$, qi $\operatorname{sun}^{4}$, Zhong-jing $\mathrm{CaO}^{2}$, and Jian $\mathrm{Ma}^{2}$ \\ ${ }^{1}$ Fuwai Hospital, National Center for Cardiovascular Diseases, Chinese Academy of Medical \\ Sciences and Peking Union Medical College, Beijing, China, State Key Laboratory of \\ Cardiovascular Disease \\ ${ }^{2}$ Fuwai Hospital, National Center for Cardiovascular Diseases, Chinese Academy of Medical \\ Sciences and Peking Union Medical College, Beijing, China \\ ${ }^{3}$ State Key Laboratory of Cardiovascular Disease, Arrhythmia Center, Fuwai Hospital, \\ National Center for Cardiovascular Diseases, Chinese Academy of Medical Sciences and \\ Peking Union Medical College \\ ${ }^{4}$ Chinese Academy of Medical Sciences and Peking Union Medical College
}

September 22, 2020

\begin{abstract}
BACKGROUND Several methods have been proposed to locate the conduction gap on the pulmonary vein isolation (PVI) ablation line. However, the value of the interval of far-field potential (FF potential) and pulmonary vein potential (PV potential) is unknown. METHODS: The state of PV potential and the location of that ablation sites were particularly recorded for analysis once LSPV was isolated in a development cohort of consecutive patients undergoing PVI ablation at a single center. These findings informed the development of a novel algorithm to discriminate the location of a conduction gap between upper portion and inferior portion of LSPV. The performance of this novel algorithm was prospectively tested in a validation cohort of consecutive re-do patients undergoing PVI ablation RESULTS: A total one hundred sixteen patients were recruited, of which 56 formed the validation cohort. Interval of FF potential and PV potential was associated with the location of the conduction gap, and the interval $<5 \mathrm{~ms}$ predicted that there was a conduction gap in the upper portion of ostium with $92.9 \%$ sensitivity and $96.9 \%$ specificity. In the prospective evaluation, the interval was able to correctly predict the site of successful ablation in 89.6\%. CONCLUSIONS: Interval of far-field potential and pulmonary vein potential is a novel and accurate marker to predict the location of a conduction gap.
\end{abstract}

\section{BACKGROUND}

Pulmonary vein isolation (PVI) is the cornerstone of catheter ablation in majority of patients with atrial fibrillation $(\mathrm{AF})^{[1,2]}$. However, non-isolation after initial encircling of the pulmonary veins (PVs) during observation on the table or discharge is not rare. Touch-up lesions are frequently required to eliminate the conduction gaps $^{[3]}$. In repeat $\mathrm{AF}$ ablation procedure, looking for conduction gaps is a critical and sometimes difficult procedure, due to the difficulty in identifying left atrium (LA)-PV gaps. Prediction of the location of conduction gaps before the ablation becomes significant for less lesion and operation time.

The aims of this study were to first, systematically evaluate the use of the interval of far-field potential (FF potential) and pulmonary vein potential (PV potential) on Lasso catheter in identifying conduction 
gap, second, to develop a novel algorithm incorporating these findings, and finally, this algorithm would be validated its efficacy for locating the conduction gap.

\section{Methods}

This study was divided into in 2 phases: (1) a development and hypothesis-generating phase whereby patients' electrophysiology study and Carto data were systemically recorded and analyzed during isolating left superior pulmonary vein (LSPV) to create a more precise algorithm to determine the location of conduction gap; and (2) a prospective validation of this new algorithm.

The study was approved by the institutional review board of Fuwai Hospital, National Center for Cardiovascular Diseases, Chinese Academy of Medical Sciences and Peking Union Medical College.

\section{Patient Selection}

Consecutive patients with a conduction gap after initial circumferential pulmonary vein ablation of LSPV or reconnection during observation on the table were enrolled. And then another consecutive repeat ablation cohort whose LA-PV of LSPV reconnected were recruited to verify the results. Patients with structural heart disease, AF on the table and common PVs confirmed by CT were excluded. Informed consent was obtained before procedure.

\section{Ablation, Electrophysiology Study and 3-dimensional electroanatomic mapping Recording}

Electrophysiology Study (EPS) and 3-dimensional electroanatomic mapping will be real-time monitored during the ablation. A 3.5-mm-tip catheter (ThermoCool Navi-Star, Biosense Webster Inc) was used to 3-dimensional electroanatomic mapping of the left atrium and ablation. Then, selective venography and focal potential will be used to determine each PV ostium, and tagged on the 3-dimensional electroanatomic map. A Lasso (Biosense-Webster Inc), the circular mapping catheters were placed inside the PV ostium to observe the change of PV potential and verify PVI during radiofrequency ablation. The power output was titrated to $20-35 \mathrm{~W}$ with a maximum target temperature of $43^{\circ} \mathrm{C}$ and an infusion rate of 17 to $25 \mathrm{~mL} / \mathrm{min}$. The end point was defined as the absence of any PV potential recorded on Lasso catheter placed within the PV ostium at least 30 minutes after PV isolation during sinus rhythm (SR). Once LSPV was isolated, the state of PV potential and the location of that ablation sites would be particularly marked for analysis.

In the validation cohort, 3-dimensional electroanatomic mapping of the left atrium would be also reconstructed first, and the state of PV potential of LSPV would be recorded and then the location of conduction gaps would be mapped. Ablation will be performed where the earliest potential demonstrated on the Lasso catheter.

\section{Definitions}

According to anatomical structure, the ostium of LSPV was divided to 2 sections: upper portion and inferior portion. In detail, upper portion referred to 9-3 o'clock direction of ostium while the inferior portion, the remain section. We also define the 10-2 o'clock direction of ostium as the roof.

Interval of far-field potential and pulmonary vein potential on Lasso catheter was the time from the offset of far-field potential to the onset of pulmonary vein potential (Figure 1). If the two potential were overlapping, and hard to identify the offset and onset of potential, the interval was therefore defined as $0 \mathrm{~ms}$.

\section{Statistical Analysis}

Continuous variables are expressed as mean $\pm 1 \mathrm{SD}$ and were analyzed by Student t test. Chi-square test Logistic regression would be used to determine factors associated with the location of conduction gaps. A receiver operating characteristic (ROC) curve analysis was performed for sensitivity and specificity analysis, with accuracy being measured by the area under the curve (AUC) of the ROC with a cutoff value represented the optimal balance between sensitivity and specificity. All statistics were performed using SPSS statistical software (release 19.0; SPSS Inc, Chicago, IL). P value [?]0.05 was considered statistically significant. 


\section{Results}

\section{Development of the Novel Algorithm}

In the development phase, sixty patients with atrial fibrillation who underwent PVI ablation in Fuwai Hospital from July 2015- July 2017 were enrolled to the development cohort. The last site was identified according to PVI in Upper and Inferior portion in 32 (53.3\%) and 28 (46.7\%) patients, respectively. In Upper portion, most of conduction gaps (30/32) located at the roof. Within the Inferior portion, all the conduction gaps located at the junction of PV. There were no significant differences in monofactor analysis among the age, gender, left atrial diameter and CHA2DS2-VASc between 2 groups (Table 1). In the logistic regression analysis, interval of FF potential and PV potential was associated with the location of the conduction gap $(\mathrm{OR}=1.550,95 \% \mathrm{CI} 1.131-2.125, \mathrm{P}=0.006)$, which indicates that the interval of the inferior portion is greater than the upper. ROC analysis of the interval was shown in Figure 2. The AUC was 0.958, and the interval $<5 \mathrm{~ms}$ predicted that there was a conduction gap in upper portion of ostium with $92.9 \%$ sensitivity and $96.9 \%$ specificity. On basis of the above findings, a novel algorithm was developed.

\section{Validation of Novel Algorithm Incorporating Interval Index}

In the validation cohort, 56 patients from September 2017 and September 2019 were recruited and formed the validation cohort. The conduction gap was found only at the inferior portion in 20 , while at the upper portion in 28 , as well as at the both portions in 8 . Scatter plot of cutoff value of interval of far-field potential and pulmonary vein potential in the validation cohort was showed in Figure3 The interval which the conduction gap was found only at inferior portion was greater than $5 \mathrm{~ms}$ in 18 of 20 patients, while the interval index was greater than $5 \mathrm{~ms}$ in 3 of 28 at upper portion. The interval was able to correctly predict the site of successful ablation in $89.6 \%$ (43 of 48 ), with $92.6 \%$ sensitivity and $85.7 \%$ specificity. An example of conduction gap that was correctly predicted by the interval in a re-do patient is shown in Figure 4. It is also interesting to find that the interval which conduction gaps were found at the both portions were all smaller than $5 \mathrm{~ms}$. After closing the conduction gap(s) at the upper portion, a new conduction gap was identified with a delay in the timing $(22.3+-13.0 \mathrm{~ms})$, which were all finally eliminated at the inferior portion.

\section{Discussion}

\section{Major Findings}

The major finding of this study is that the interval of FF potential and PV potential of LSPV allowed for more accurate location of the conduction gap. The key findings in our study are as follows:(1) upper portion of LSPV is the dominate conduction of LSPV. (2)the interval $<5$ ms predicted that there was a conduction gap in upper portion of ostium $92.9 \%$ sensitivity and $96.9 \%$ specificity.

\section{The Possible Anatomic Considerations}

Understanding the anatomic relationship between the left atrial appendage (LAA) and LSPV as well as the cardiac conduction system is key to locate the conduction gaps. LAA grows in the front of LSPV, with a ridge in the middle, so there is the reason why Lasso can record an another far-field potential except for PV potential within ostium of LSPV.

In normal, Bachmann's bundle (BB, Figure5) connects with sinus node of right atrium, and branches to left atrium and passes around the neck of the left atrial appendage, reuniting to continue into the musculature of the lateral and posteroinferior atrial walls. ${ }^{[4]}$ Therefore, As for LSPV, the earliest breakthrough occurs at the roof and then the impulse goes down anteriorly and posteriorly to the inferior portion. When LSPV was also activated starting from the upper portion, LAA was also activated at the same time, so that Far-field potential and PV potential were overlapped without any intervention generally. When the upper portion was block out, especially the roof, but with a conduction gap at the inferior portion, LSPV would be activated from inferior portion firstly, and the route that electrical activity introduced to LSPV would be prolonged. As a result, PV potential and far-field potential would be separated. Conversely, if the upper portion was not blocked out, the route that electrical activity introduces to LSPV would not be changed. Therefore, relative 
position of PV potential and far-field potential would not change a lot. This conformed to our studies that the interval of $\mathrm{PV}$ potential and far-field potential $<5 \mathrm{~ms}$ predicted that there was a conduction gap in upper portion.

\section{Strategy for locating conduction gaps}

It is worth noting that Miyamoto K, etc have reported that when there are more than 2 gaps with different conduction times over the gaps, conduction over a gap with a long conduction time was frequently masked by another gap with a short conduction time ${ }^{[5]}$. In our study, we can see the 9 patients with conduction gaps in both portions whose intervals are still smaller than 5ms. After eliminating the upper portion ones, the interval suddenly prolonged a lot and finally closed the residual gaps at inferior portion. It can be seen that the upper portion is dominate conduction of LSPV. Thus, on basis of the above findings, a novel algorithm was developed (Figure 6). Meeting a LSPV after circumferential pulmonary vein ablation but non-isolation or reconnection, we can firstly check the interval to know where should be touched-up. If the interval is larger than $5 \mathrm{~ms}$, we should first exclude the conduction gap locating at the upper portion, especially at the roof. While the interval is smaller the $5 \mathrm{~ms}$, the conduction gap was supposed to be locating at upper portion at first. And if PV potential delay was found after eliminating the upper conduction gap, another conduction gap should be considered at inferior portion.

\section{Comparison with Previous Studies}

Although Experience operators routinely achieve 100\% acute PVI rates using standard mapping techniques, reconnection between PV and atrium during observation on the table or discharge is not rare ${ }^{[6,7]}$. Previous studies have illustrated that several methods Guided by Nav $\mathrm{X}^{[5]}$, Rhythmia ${ }^{[8]}$, and CARTO mapping system $^{[9]}$ allowed for an acquisition of multiple $3 \mathrm{D}$ anatomical points along with the local activation time to have visual identification of the conduction gap. However, as Miyamoto et al. ${ }^{[5]}$ demonstrated that sometimes it would be misled by low amplitude, and/or fractionated electrogram so as to made additional efforts to distinguish atrial and PV electrogram accurately. In the Benito et al. ${ }^{[10]}$ case report and in the Masuda et al. ${ }^{[8]}$ study, the voltage map failed to identify gap localization in two-thirds of the cases. What's more, as for Cryoballoon ablation, mapping electrode 'Achieve', with large electrode spacing and unfixed shape, makes it more difficult to accurately locate the conduction gap during cryoablation. Hence, the combined use of the interval could facilitae to recognize whether the roof we have frozen and the location of conduction gap for non-isolation after initial encircling and electrical reconnection quickly and simply.

\section{Study Limitations}

This study has several limitations. First, the sample size in prospective cohort is relatively small and should be further validated in larger populations with multiple centers. Second, although the new algorithm could accurately distinguish upper portion from inferior portion of conduction gap, it does not localize to specific location. Furthermore, this new algorithm is just applicable to LSPV, other PV should be further researched.

\section{Conclusions}

Interval of far-field potential and pulmonary vein potential is a novel and accurate marker to predict the location of a conduction gap.

\section{Funding}

This work was supported by a grant of National Natural Science Foundation of China (\#81670309).

\section{Acknowledgments}

Conflict of interests: None

1. Haissaguerre M, Jais P, Shah DC, Takahashi A, Hocini M, Quiniou G, et al. Spontaneous initiation of atrial fibrillation by ectopic beats originating in the pulmonary veins. The New England journal of medicine 1998; 339(10):659-666. 
2. Ouyang F, Bansch D, Ernst S, Schaumann A, Hachiya H, Chen M, et al.Complete isolation of left atrium surrounding the pulmonary veins: new insights from the double-Lasso technique in paroxysmal atrial fibrillation . Circulation 2004; 110(15):2090-2096.

3. Sandorfi G, Rodriguez-Manero M, Saenen J, Baluja A, Bories W, Huybrechts W, et al. Less Pulmonary Vein Reconnection at Redo Procedures Following Radiofrequency Point-by-Point Antral Pulmonary Vein Isolation With the Use of Contemporary Catheter Ablation Technologies .JACC Clin Electrophysiol 2018; 4(12):1556-1565.

4. Ho SY, Ernst S. Anatomy for Cardiac Electrophysiologists: A Practical Handbook . Cardiotext Publishing; 2012.

5. Miyamoto K, Tsuchiya T, Yamaguchi T, Nagamoto Y, Ando S, Sadamatsu K, et al. A new method of a pulmonary vein map to identify a conduction gap on the pulmonary vein antrum ablation line . Circ J 2011; 75(10):2363-2371.

6. Ciconte G, Mugnai G, Sieira J, Velagić V, Saitoh Y, Irfan G, et al.On the Quest for the Best Freeze: Predictors of Late Pulmonary Vein Reconnections After Second-Generation Cryoballoon Ablation .Circ Arrhythm Electrophysiol 2015; 8(6):1359-1365.

7. Park CI, Lehrmann H, Keyl C, Weber R, Schiebeling J, Allgeier J, et al. Mechanisms of pulmonary vein reconnection after radiofrequency ablation of atrial fibrillation: the deterministic role of contact force and interlesion distance. J Cardiovasc Electrophysiol 2014; 25(7):701-708.

8. Masuda M, Fujita M, Iida O, Okamoto S, Ishihara T, Nanto K, et al.The identification of conduction gaps after pulmonary vein isolation using a new electroanatomic mapping system . Heart Rhythm 2017; 14(11):1606-1614.

9. Dong J, Liu X, Long D, Yu R, Tang R, Lu F, et al.Single-catheter technique for pulmonary vein antrum isolation: is it sufficient to identify and close the residual gaps without a circular mapping catheter? J Cardiovasc Electrophysiol 2009; 20(3):273-279.

10. KOSIUK J, HILBERT S, HINDRICKS G, BOLLMANN A. Channel-Based Gap Mapping for Pulmonary Vein Isolation. Journal of Cardiovascular Electrophysiology 2016; 27(4):488-489.

Table1.Baseline Characteristics of Patients in Development and Prospective Cohort

\begin{tabular}{|c|c|c|c|c|c|c|c|c|c|c|}
\hline & $\begin{array}{l}\text { Developn } \\
\text { Co- } \\
\text { hort }\end{array}$ & $\begin{array}{l}\text { nEtvelopm } \\
\text { Co- } \\
\text { hort }\end{array}$ & $\begin{array}{l}\text { nEdtvelopm } \\
\text { Co- } \\
\text { hort }\end{array}$ & $\begin{array}{l}\text { AEdvelo } \\
\text { Co- } \\
\text { hort }\end{array}$ & $\begin{array}{l}\text { nErrts } \\
\text { Co- } \\
\text { hort }\end{array}$ & $\begin{array}{l}\text { iPerospecti } \\
\text { Co- } \\
\text { hort }\end{array}$ & $\begin{array}{l}\text { Perospecti } \\
\text { Co- } \\
\text { hort }\end{array}$ & $\begin{array}{l}\text { iPerospecti } \\
\text { Co- } \\
\text { hort }\end{array}$ & $\begin{array}{l}\text { Perospecti } \\
\text { Co- } \\
\text { hort }\end{array}$ & \\
\hline & All & $\begin{array}{l}\text { Upper } \\
\text { Por- } \\
\text { tion }\end{array}$ & $\begin{array}{l}\text { Inferior } \\
\text { Por- } \\
\text { tion }\end{array}$ & $\begin{array}{l}\mathbf{P} \\
\text { Value }\end{array}$ & & All & $\begin{array}{l}\text { Upper } \\
\text { Por- } \\
\text { tion }\end{array}$ & $\begin{array}{l}\text { Inferior } \\
\text { Por- } \\
\text { tion }\end{array}$ & $\begin{array}{l}\text { Both } \\
\text { Por- } \\
\text { tion }\end{array}$ & $\begin{array}{l}\text { Both } \\
\text { Por- } \\
\text { tion }\end{array}$ \\
\hline $\begin{array}{l}\text { Patients, } \\
\mathbf{n}(\%)\end{array}$ & 60 & $32(53)$ & $28(47)$ & NA & & 56 & $28(50)$ & $20(36)$ & $8(14)$ & $8(14)$ \\
\hline $\begin{array}{l}\text { Male, } \\
\text { n(\%) }\end{array}$ & $47(78)$ & $25(78)$ & $22(79)$ & .967 & & $49(88)$ & $24(86)$ & $17(85)$ & $8(100)$ & $8(100)$ \\
\hline $\begin{array}{l}\text { Age, } \\
\text { y }\end{array}$ & $56.3 \pm 8.4$ & $57.1 \pm 7.8$ & $55.3 \pm 9.0$ & .419 & & $54.1 \pm 9.7$ & $56.2 \pm 10.1$ & $153.2 \pm 9.3$ & $49.1 \pm 7.7$ & $49.1 \pm 7.7$ \\
\hline $\begin{array}{l}\text { LA, } \\
\text { mm }\end{array}$ & $37.1 \pm 6.0$ & $36.2 \pm 4.7$ & $36.5 \pm 4.7$ & .892 & & $37.0 \pm 3.5$ & $37.7 \pm 3.7$ & $37.6 \pm 3.3$ & $37.6 \pm 3.1$ & $37.6 \pm 3.1$ \\
\hline $\begin{array}{l}\text { CHA2DS } \\
\text { VASc }\end{array}$ & $21.6 \pm 1.7$ & $1.9 \pm 1.8$ & $1.2 \pm 1.5$ & .095 & & $1.3 \pm 1.5$ & $1.3 \pm 1.4$ & $1.7 \pm 1.7$ & $0.3 \pm 0.7$ & $0.3 \pm 0.7$ \\
\hline
\end{tabular}

LA indicates left atrial diameter 
Figure 1. Interval of far-field potential and pulmonary vein potential on Lasso catheter measured from the offset of far-field potential to the onset of pulmonary vein potential.

A. Far-field potential and PV potential were overlapped, so the interval between them was defined as 0ms.

B. Far-field potential and PV potential were separated, so the interval between them was $46 \mathrm{~ms}$.

CS, coronary sinus; L1.2, Lasso catheter distal electrode; L9.10, Lasso catheter proximal electrode

Figure 2. Receiver operating characteristic analysis for different Interval of far-field potential and pulmonary vein potential.

Figure 3. Scatter plot of cutoff value of interval of far-field potential and pulmonary vein potential in the validation cohort.

Figure 4. An example of conduction gap that was correctly predicted by the interval in a re-do patient.

A. Lasso was placed within the ostium of LSPV, PV and atrium were found reconnected. The interval between FF potential and PV potential was $0 \mathrm{~ms}$.

B. Pacing at distal CS catheter to distinguish and confirm the existence of PV potential.

C. The successful ablation target was only located at the roof of LSPV. Blue point represents the ablation target, white point represents pulmonary vein ostium and pink point represents the mitral valve.

LSPV indicates left superior pulmonary vein; LIPV, left inferior pulmonary vein; RSPV, right superior pulmonary vein; RIPV, right inferior pulmonary vein; PV, pulmonary vein ; LAA, left atrial appendage; CS, coronary sinus; FF potential, far-field potential. L1.2, Lasso catheter distal electrode

L9.10, Lasso catheter proximal electrode

Figure 5. Bachmann's bundle branches to left atrium and passes around the neck of the left atrial appendage, reuniting to continue into the musculature of the lateral and posteroinferior

atrial walls. Red point represents pulmonary vein ostium. LSPV indicates left superior pulmonary vein; RSPV, right superior pulmonary vein; LAA, left atrial appendage;

Figure 6. Diagnostic stepwise for location conduction gap(s). 


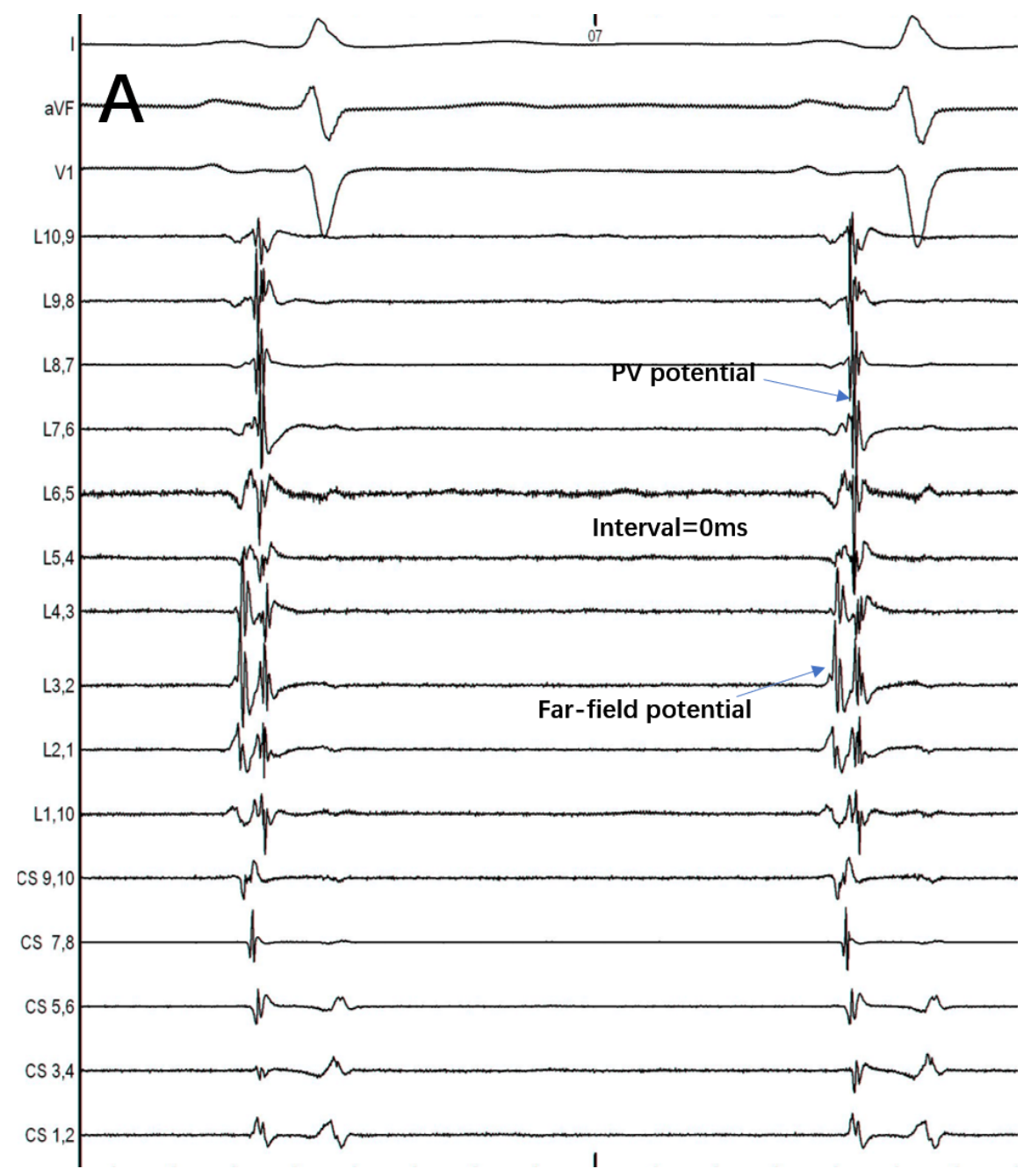




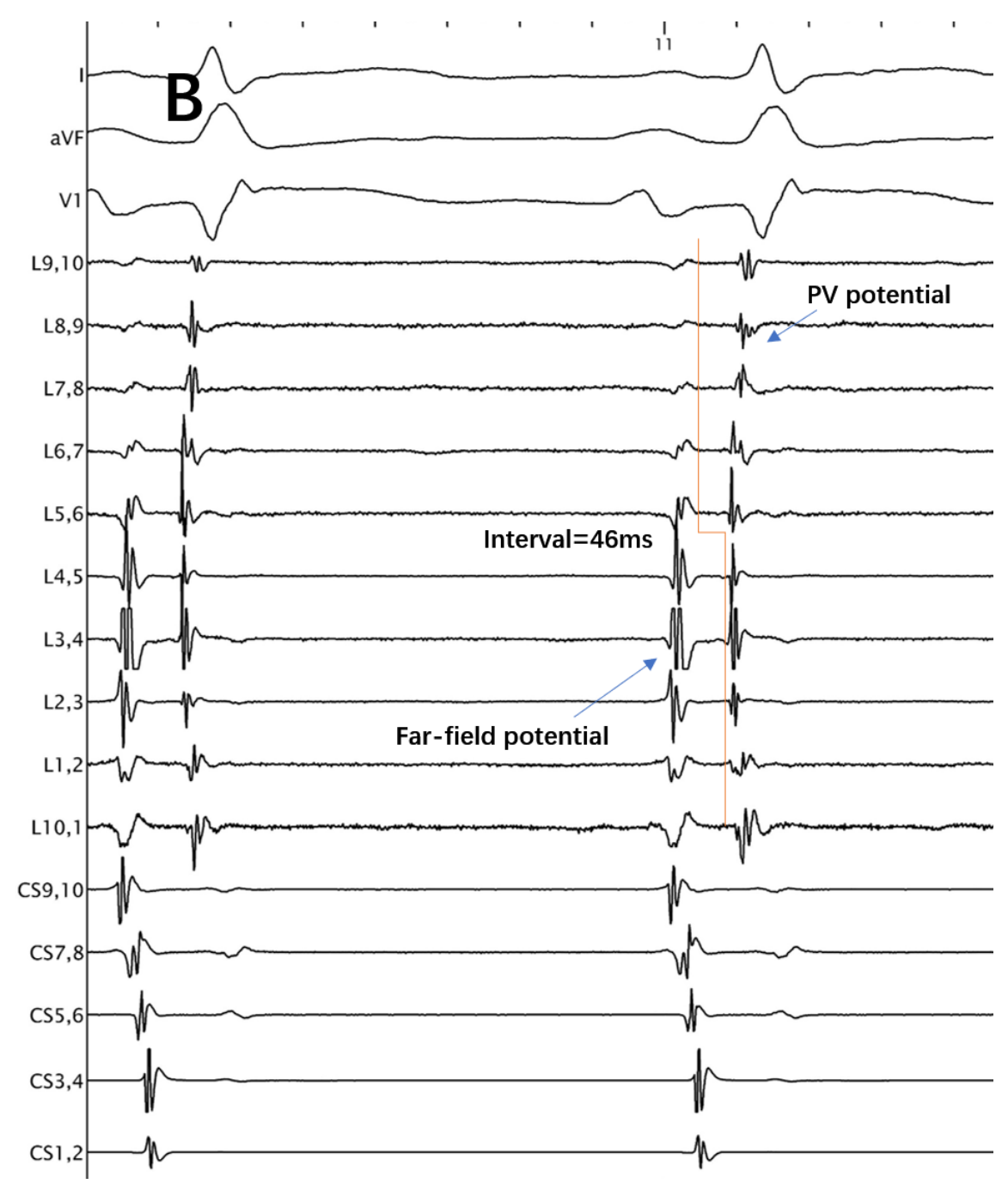



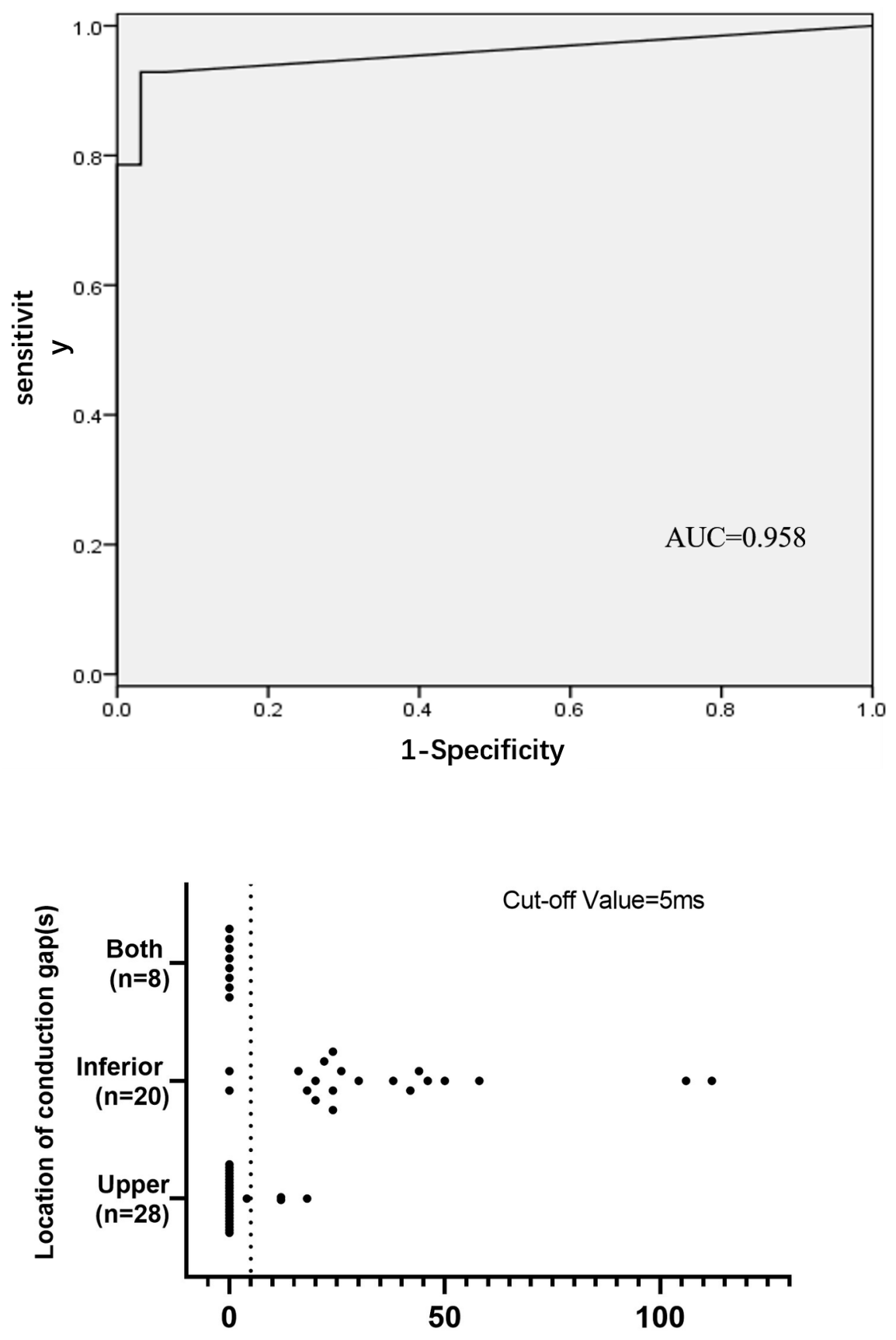

Interval of far field potential and pulmonary vein potential(ms) 

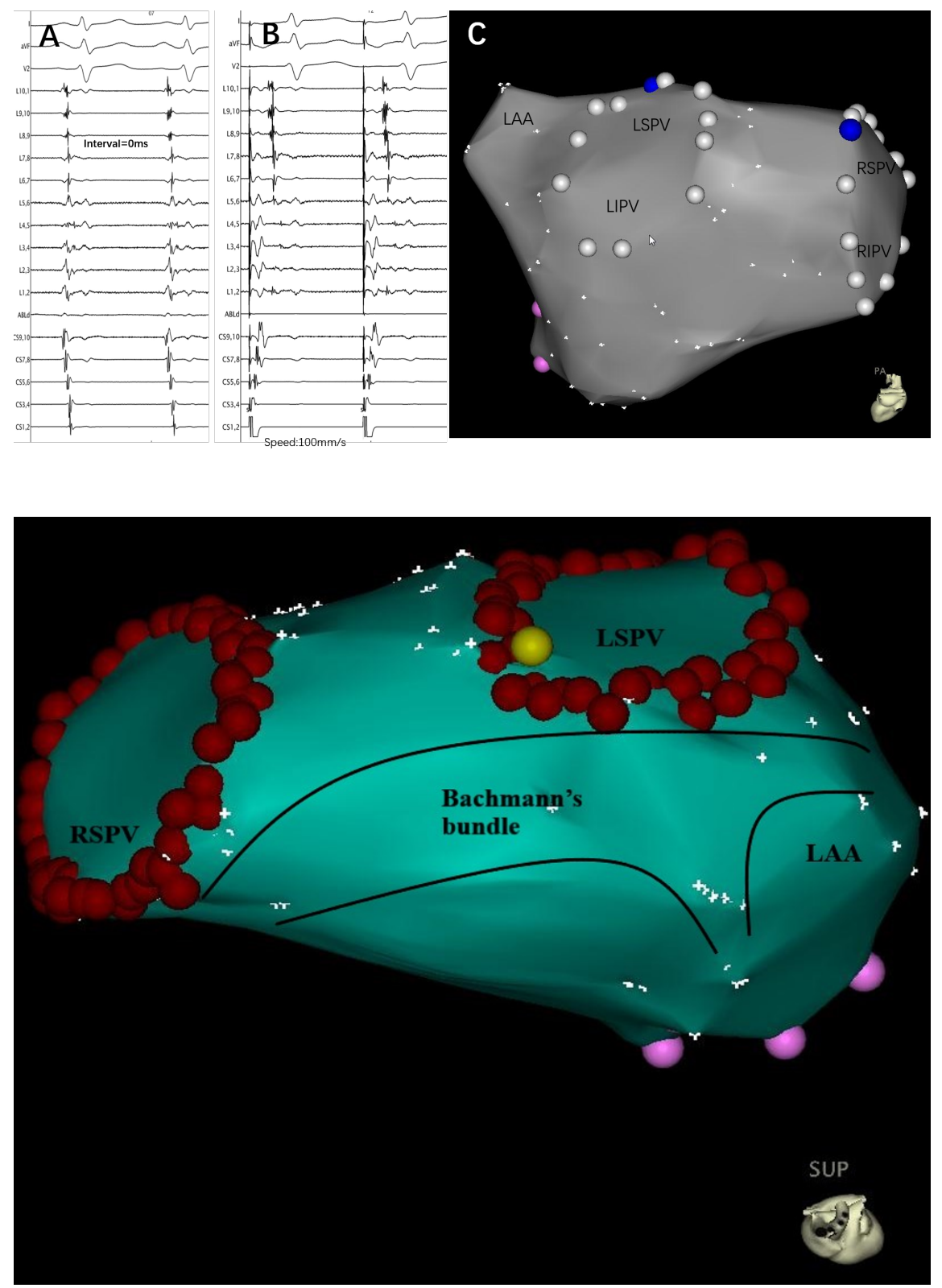


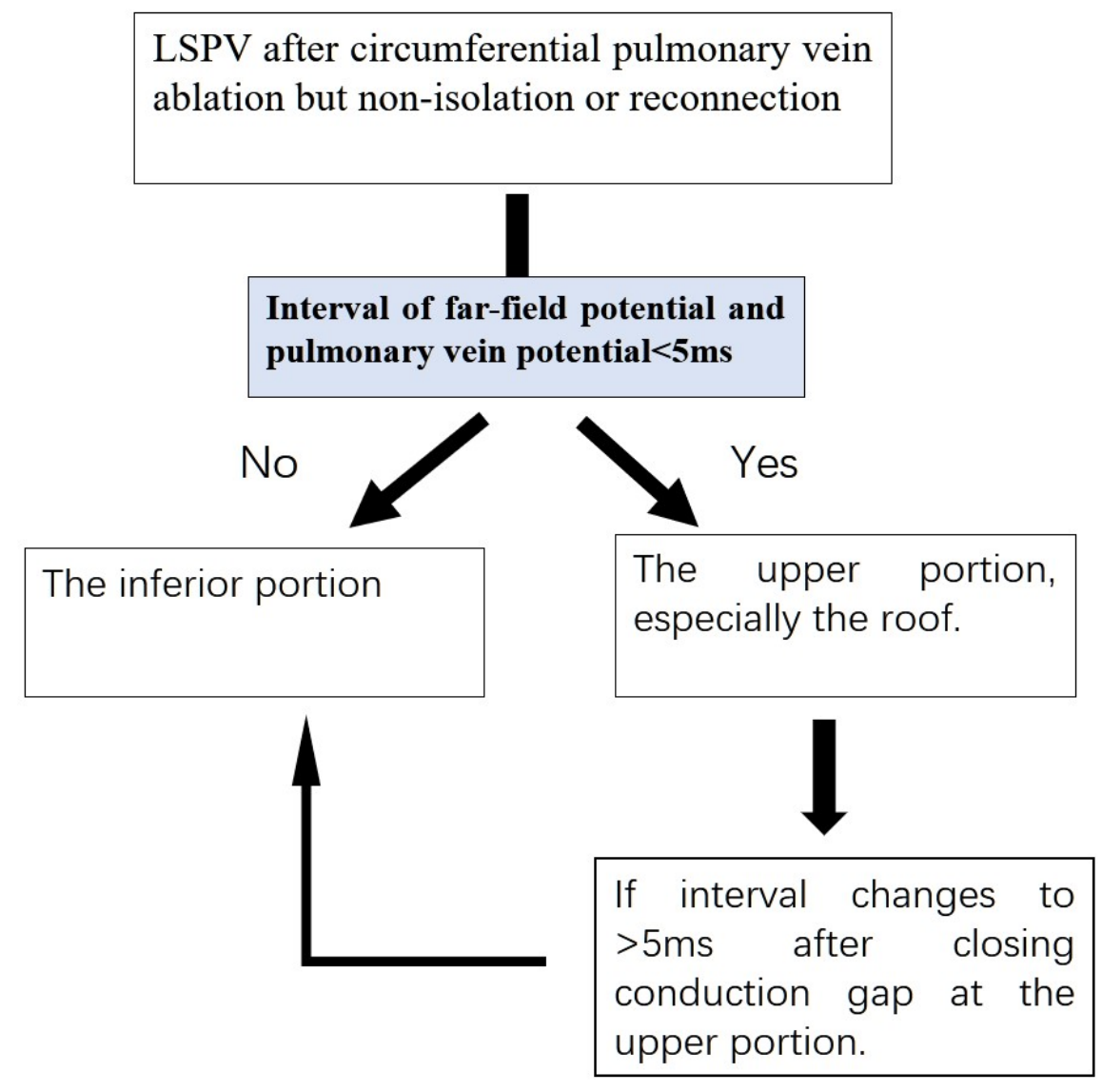

